\title{
DESCRIÇÃO DAS PROPRIEDADES FÍSICAS DAS SUPERFÍCIES PT(100) E PT(111) UTILIZANDO SIMULAÇÕES COMPUTACIONAIS BASEADAS NA TEORIA DO FUNCIONAL DA DENSIDADE
}

\author{
Description of the Physical Properties of Pt (100) and Pt (111) Surfaces Using \\ Computational Simulations based on Functional Theory of Density
}

Descripción de las Propiedades Físicas de las Superficies Pt (100) y Pt (111)

Utilizando Simulaciones Computacionales basadas en la Teoría del Funcional de la Densidad

\section{Eduardo Dias R. Sousa ${ }^{1}$, Regina Lelis-Sousa ${ }^{* 1}$}

${ }^{1}$ Curso de Física e Laboratório de Pesquisa em Materiais para Aplicações em Dispositivos Eletrônicos, Universidade Federal do Tocantins, Campus de Araguaína, Unidade Cimba, TO, Brasil.

*Correspondência: Laboratório de Pesquisa em Materiais para Aplicações em Dispositivos Eletrônicos (LABMADE), Universidade Federal do Tocantins, Av. Paraguai, s/n, Esquina com Uxiramas, Setor Cimba, Araguaína, Tocantins, Brasil. CEP:77.824-838.e-mail rlsousa@uft.edu.br.

\section{RESUMO}

Cristal de platina e suas superfícies são de grande interesse porque utilizadas em diferentes áreas tecnológicas e, recentemente, têm se destacado como catalisadores em células a combustível. Todavia, problemas desafiadores precisam ser sobrepujados para que a eficiência em geração de energia seja satisfatória e permita o uso em larga escala. A modelagem computacional é ferramenta útil para responder a questões fundamentais relativas aos processos de catálise nos dispositivos eletroquímicos. Apresenta-se aqui uma investigação sistemática de propriedades estruturais, energéticas e eletrônicas do cristal e das superfícies de $\operatorname{Pt}(100)$ e $\operatorname{Pt}(111)$ empregando metodologia de primeiros princípios baseada na Teoria do Funcional da Densidade. Utilizou-se técnicas de espaço recíproco que permitiram a descrição da periodicidade destes sistemas aliada a um baixo custo computacional. A convergência de parâmetros de cálculos que devem ser ajustados para sistemas metálicos é discutida em detalhes. Estrutura cristalina, energia de coesão e propriedades eletrônicas para o cristal de Pt estão em excelente acordo com os dados experimentais e teóricos da literatura. Propriedades estruturais, energéticas e eletrônicas das superfícies Pt(100) e $\operatorname{Pt}(111)$ são discutidas. Testes de convergência evidenciaram ser imprescindível o uso de fatias com pelo menos 06 camadas atômicas para a correta descrição computacional destas supefícies. A comparação com simulações computacionais da literatura ou resultados experimentais mostram que a metodologia é adequada à descrição da Pt e suas superfícies e o resultados apresentados podem ser úteis para nortear futuras simulações computacionais de sistemas mais complexos envolvendo este material.

Palavras-chave: Superfícies de Platina, Teoria do Funcional da Densidade, Estrutura Eletrônica.

\section{ABSTRACT}

Pt crystal and its surfaces are of great interest because they are used in different technological areas and have been used as a catalysts in fuel cells. However, challenging problems need to be overcome because the efficiency on energy conversion is not satisfactory yet and do not allow a large-scale use for these electrochemical devices. In this way, computational modeling is a useful tool to answer fundamental questions regarding catalysis processes in electrochemical devices. Here we present a systematic investigation of the structural, energetic and electronic properties of the Pt crystal and Pt (100) and Pt (111) surfaces employing first principles methodology based on 
Density Functional Theory. We used reciprocal space techniques that allowed us to describe the periodicity of these systems together with a low computational cost. The convergence of parameters of the calculations which must be adjusted for metallic systems is discussed in detail. Crystalline structure, cohesion energy and electronic properties for Pt crystal are in excellent agreement with experimental and theoretical data in the literature. Structural, energetic and electronic properties of Pt(100) and Pt(111) surfaces are discussed. Convergence tests evidenced that it is essential to use slabs with at least 06 atomic layers for the correct computational description of these surfaces. The comparison with published computational simulations on the literature or experimental results show that our methodology is adequate to the description of Pt crystal and its surfaces and the presented results can be useful to guide future computational simulations of more complex systems involving this material.

Keywords: Pt metallic surface, Density Functional Theory, Electronic structure.

\section{RESUMEN}

El cristal Pt y sus superficies son de gran interés porque se utilizan en diferentes áreas tecnológicas y se han utilizado como catalizadores en las pilas de combustible. Sin embargo, es necesario superar problemas difíciles porque la eficiencia en la conversión de energía no es satisfactoria todavía y no permiten un uso a gran escala para estos dispositivos electroquímicos. De esta manera, el modelado computacional es una herramienta útil para responder a preguntas fundamentales sobre los procesos de catálisis en dispositivos electroquímicos. Aquí presentamos una investigación sistemática de las propiedades estructurales, energéticas y electrónicas del cristal Pt y superficies Pt (100) y Pt (111) empleando la metodología de los primeros principios basada en la Teoría Funcional de la Densidad. Utilizamos técnicas espaciales recíprocas que nos permitieron describir la periodicidad de estos sistemas junto con un bajo costo computacional. La convergencia de parámetros de los cálculos que deben ser ajustados para sistemas metálicos se discute en detalle. La estructura cristalina, la energía de cohesión y las propiedades electrónicas para el cristal de Pt están en excelente acuerdo con los datos experimentales y teóricos de la literatura. Se discuten las propiedades estructurales, energéticas y electrónicas de las superficies Pt (100) y Pt (111). Las pruebas de convergencia evidenciaron que es esencial usar losas con al menos 06 capas atómicas para la descripción computacional correcta de estas superficies. La comparación con las simulaciones computacionales publicadas en la literatura o los resultados experimentales muestra que nuestra metodología es adecuada a la descripción del cristal Pt y sus superficies y los resultados presentados pueden ser útiles para orientar futuras simulaciones computacionales de sistemas más complejos que involucren este material.

Descriptores: Superficies de Platino, Teoría del Funcional de la Densidad, Estructura Electrónica.

\section{INTRODUÇÃO}

Superfícies metálicas têm sido extensivamente estudadas porque são fundamentais em muitas aplicações tecnológicas. Em especial, superfícies de Pt e Pd e suas nanoestruturas têm recebido especial atenção porque utilizadas como catalisador em células eletroquímicas ou outros dispositivos que permitem conversão de energia. $\mathrm{O}$ estado da arte nesta área de pesquisa é obter soluções para maior eficiência da catálise. Um exemplo são as células a combustível de etanol também denominadas de Direct Alcohol Fuel Cells, ou ainda DEFC - Direct Ethanol Fuel Cell
(CHUNG et al., 2014, LOPES., 2013; WANG; LIU, 2008). Estes são basicamente, dispositivos eletroquímicos que convertem a energia química de um combustível e de um oxidante diretamente em corrente elétrica (WENDT; GöTZ; LINARDI., 2000). Em geral, têm estruturas baseadas em um reservatório de combustível, um anodo, um eletrólito e um catodo. Comumemente, o anodo é formado por um metal, e, neste caso, a Pt tem sido amplamente utilizada porque a reação anódica precisa ser catalisada (LOPES., 2013). A reação de oxidação envolve muitos passos intermediários e a cinética é extremamente complexa. 
É crucial haver quebra das ligações C--C, mas neste processo diferentes caminhos intermediários se tornam acessíveis e todos eles dependem da superfície do catalisador (metais nanoestruturados, diferentes superfícies metálicas, ...), da produção de substâncias que envenenam o catalisador (indisponibiliza os sítios catalíticos) (CHUNG et al., 2014, WANG; LIU, 2008) e de outros fatores que não serão discutidos aqui porque fora do escopo do presente artigo. Em uma DEFC, um dos principais venenos do catalisador é o CO (óxido de carbono) e sua produção devem ser evitadas uma vez que diminuem drasticamente os sítios catalíticos, bloqueando a reação de oxidação e impedindo a formação do $\mathrm{CO}_{2}$ (LOPES., 2013).

A simulação computacional ab-initio tem sido uma ferramenta útil para modelar as células a combustível e, por meio de um melhor entendimento microscópico destes sistemas, propor soluções que conduzam ao aumento da eficiência catalítica. Esforços científicos substanciais têm sido empreendidos nos últimos anos e a quantidade de trabalhos com este foco têm crescido. Em geral, a Teoria do Funcional da Densidade tem sido a ferramenta mais utilizada (LI et al, 2016; ZHAO et al, 2015; SHENG et al, 2014; SKÚLASON et al, 2014; KORTLEVER, R., 2015).

Neste artigo, tem-se por objetivo investigar as propriedades estruturais, energéticas e eletrônicas das superfícies de Pt(100) e Pt(111) com metodologia de primeiros princípios baseada na Teoria do Funcional da Densidade em conjunto com o uso de pseudopotenciais e funções de base tipo ondas planas. A escolha destas ferramentas se justifica porque é possível descrever adequadamente a periodicidade bidimensional das superfície de Pt, que é crucial na descrição dos processos de catálise. Ocupou-se de análise minuciosa dos parâmetros a serem convergidos durante os cálculos com a finalidade de se obter a correta descrição destas superfícies, consistindo no primeiro passo para o futuro estudo das reações de oxidação do etanol catalisada por Pt.

\section{MATERIAIS E MÉTODOS}

Todos os resultados foram obtidos meio de metodologia de primeiros princípios e baseada na Teoria do Funcional da Densidade (DFT - Density Functional Theory) (HOHENBERG e KOHN, 1964; KOHN, W. SHAM, L. J. 1965) baseada no esquema de Kohn-Sham (KS) e implementada no código computacional de domínio público PWSCF (Plane Wave Self-Consisted Field) da distribuição QuantumEspresso (opEn Source Package for Research in Electronic Structure, Simulation, and Optimization) (GIANNOZZI et al, 2009). Empregou-se o funcional de troca e correlação GGA (GGA - Generalized Gradient Approximation) e com parametrização de Perdew-Burke-Ernzerhof, denominada PBE (PERDEW, BURK e ERNZERHOF, 1996), que foi a aproximação escolhida por fornecer excelentes resultados para energias atômicas e energias de ligação. Para maior eficiência computacional e simplicidade no tratamento de sistemas cristalinos, empregou-se funções de base tipo ondas planas (PW - Plane Waves) para os orbitais de KS em conjunto com pseudopotenciais Ultrasuaves para representação dos elétrons de caroço. Especificamente, a configuração eletrônica do pseudopotencial da Pté $5 \mathrm{~d}^{9} 6 \mathrm{~s}^{1}$. Para esta metodologia, a convergência das funções de base é simples e baseada no valor de energia de corte das ondas planas e nenhuma correção adicional é necessária. Para a integração das equações de KS dentro da Zona de Brillouin (BZ), a metodologia de Monkhorst-Pack foi empregada para gerar conjuntos de pontos k e os respectivos pesos associados. Deve-se realizar somatórios sobre todas as bandas e para cada ponto $\mathrm{k}$ calculados para temperatura nula. Nos sistemas metálicos, é necessário realizar este procedimento para um sistema onde o nível mais alto ocupado pode cruzar 
o nível de Fermi, ou seja, durante o processo de soma, valores podem assumir valor nulo (abruptamente). $\mathrm{O}$ resultado é instabilidade numérica e não convergência do SCF. Uma solução para o problema é aumentar a amostrogem do espaço recíproco, introduzindo mais pontos $\mathrm{k}$, mas aumentando o esforço computacional. Foi desenvolvida uma estratégia interessante para resolver este problema. O que se faz é suavisar a curva de distribuição de Fermi-Dirac atribuindo uma temperatura eletrônica fictícia aos elétrons, em um procedimento de "smearing" da densidade eletrônica. O processo consiste em suavizar a curva de ocupação e, como consequência, não há contribuições que "pulam" de 0 para 1 abruptamente. Empregou-se nos cálculos aqui discutidos a técnica de "smearing" de Marzari-Vanderbilt (MARZARI; VANDERBILT, 1999). E, para a implementação da metodologia, é preciso ajustar o valor de "alargamento" da função gaussiana para a integração no espaço recíproco. Este é um ponto crucial e cuidadosos testes de convergência devem ser testados a fim de garantir que os sitemas físicos de interesse são adequadamente descritos pela técnica. Nas simulações utilizando PWSCF, realizouse testes com os seguintes valores de "alargamento" das funcões gaussianas ("degauss"): para : 0,01; 0,02; 0,03; 0,04; 0,05; 0,06; 0,07; 0,08; 0,09;0,001;0,002;0,003; 0,004; 0,005; 0,006; 0,007; 0,008; 0,009; 0,0001; 0,0002; 0,0003; 0,0004; 0,0005; 0,0006; 0,0007; 0,0008; 0,0009; 0,00001; 0,00002; 0,00003; 0,00004; 0,$00005 ; 0,00006 ; 0,00007 ; 0,00008 ; 0,00009$. A escolha do valor de smearing adequado aos sistemas de $\mathrm{Pt}$ se deu em processos de relaxação estrutural nos quais o parâmetro de rede cristalino foi fixado no valor experimental de 3,92 $\AA$ (SINGH-MILLER; MARZARI, 2009), o valor de energia de corte das ondas planas (Ecut) e do corte da densidade eletrônica (Ecutrho) foram ajustados para, respectivamente, Ecut $=60$ Ry e Ecutrho $=10 *$ Ecut. $\mathrm{O}$ alto valor de Ecutrho é necessário porque pseudopotenciais ultrusuaves não têm norma garantida por construção e, em consequência, é necessário que o corte da densidade eletrônica tenha valores muito maiores que quatro vezes o valor de Ecut. Ajustou-se os critérios de convergência do procedimento SCF e de otimizações de geometria em, respectivamente, $1 \times 10^{-8}$ Ry e $1 \times 10^{-4} \mathrm{Ry} /$ Bohr, a fim de assegurar convergência numérica rigorosa. Os resultados dos testes serão apresentados posteriormente.

\section{RESULTADOS E DISCUSSÃO}

O cristal de Pt (ou Pt bulk) pertence ao sistema cúbico e se cristaliza em uma estrutura cúbica de face centrada (FCC - Face Centered Crystal), cuja célula unitária primitiva e convencional podem ser visualizadas na Fig. 1. A célula unitária primitiva tem apenas um átomo na base.

Figura 1. Representação da célula unitária do cristal de Pt. À esquerda vê-se a célula primitiva com translação de 4 unidades cristalinas e à direita, a célula convencional.
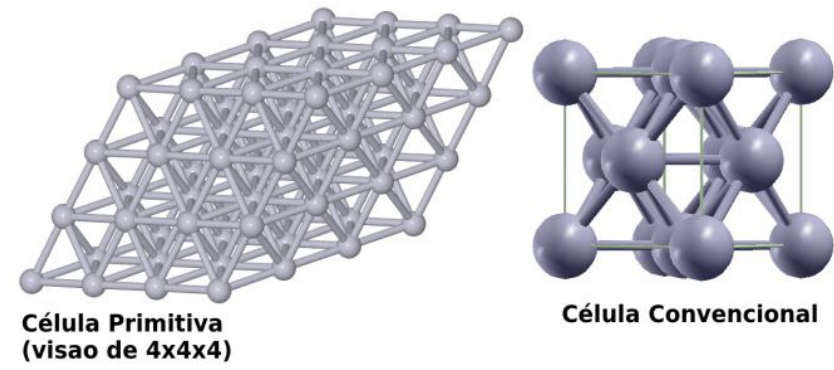

Os resultados podem ser vistos na Fig. 2. em que se analisa a variação da energia de Fermi e da energia total como função da variação do valor da largura da gaussiana utilizada na convergência do SCF (parâmetro "degauss").

Figura 2. Dados para variação da energia de Fermi e da energia de total do cristal de Pt em função da variação dos valores do parâmetro "degauss" calculados com diferentes grades

de pontos $\mathrm{k}$.

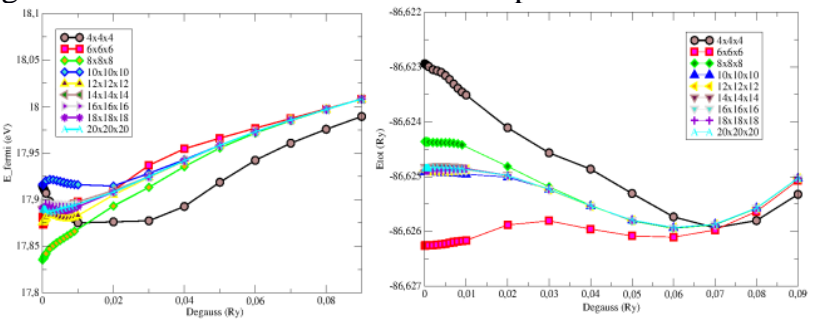


Para visualização de alguns detalhes, uma ampliação da Fig. 2 é mostrada na Fig. 3, com valores de degauss entre 0 e 0,001 Ry e a correspondente variação dos valores da Energia de Fermi.

Figura 3. Dados para variação da energia de Fermi do cristal de Pt em função da variação dos valores do parâmetro "degauss" calculados com diferentes grades de pontos k.

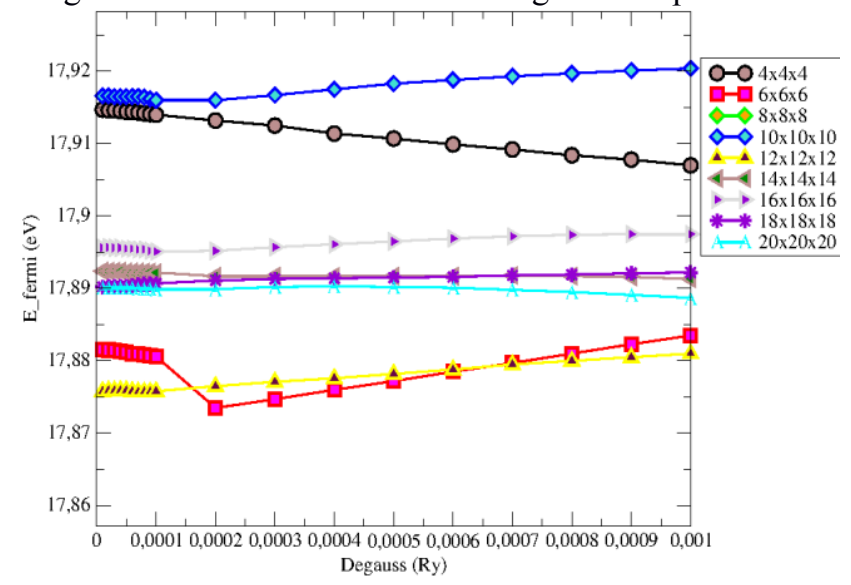

Percebe-se claramente influência do número de pontos k no processo de SCF, conforme sugerido pelo criador desta metodologia, M. Marzari (MARZARI; VANDERBILT, 1999). Em adição, os resultados mostrados nas Figs. 2 e 3, sugerem que uma grade de pontos ks obtidos com $\mathrm{k}_{\mathrm{x}} \times \mathrm{k}_{\mathrm{y}} \times \mathrm{k}_{\mathrm{z}}=12 \times 12 \times 12$ é adequada para a correta descrição do cristal de Pt. Em particular, também averiguou-se que é possível acelerar-se a convergência da grade de pontos $\mathrm{k}$ se o ponto $\Gamma$ é excluído da amostragem. $E$, finalmente, identifica-se que qualquer valor de degaus entre $10^{-5}$ Ry e 0.04 Ry nos fornece valores de Energia de Fermi que diferem entre si por menos de $0.01 \mathrm{eV}$. Assim sendo, todos os resultados posteriores terão valor de degaus ajustado para $0.01 \mathrm{eV}$ e grade de MK de 12x12x12 (excluindo o ponto Gamma).

Dando sequência aos testes de convergência, concluímos, com base na análise do comportamento da energia de Fermi e da energia total como função de Ecut (ver Fig. 4), que Ecut $=35$ Ry e Ecutrho=350 Ry são suficientes para obter convergência para a Platina bulk.
Parâmetro de rede ( $\mathbf{a}_{\mathbf{0}}$ - $\left.\mathbf{A}\right)$, energia de coesão $\left(\mathbf{E}_{\text {coesão }}-\mathbf{e V}\right)$ e também o módulo de compressibilidade volumétrica (bulk modulus - $\boldsymbol{B}_{0}$ - $\boldsymbol{G P a}$ ) para o cristal de Pt estão listados na Tab. 1. Empregou-se 03

Figura 4. Variação da energia total e da energia de Fermi do cristal de Pt em função da variação dos valores da energia de corte das ondas planas, Ecut, utilizadas como funções de base.
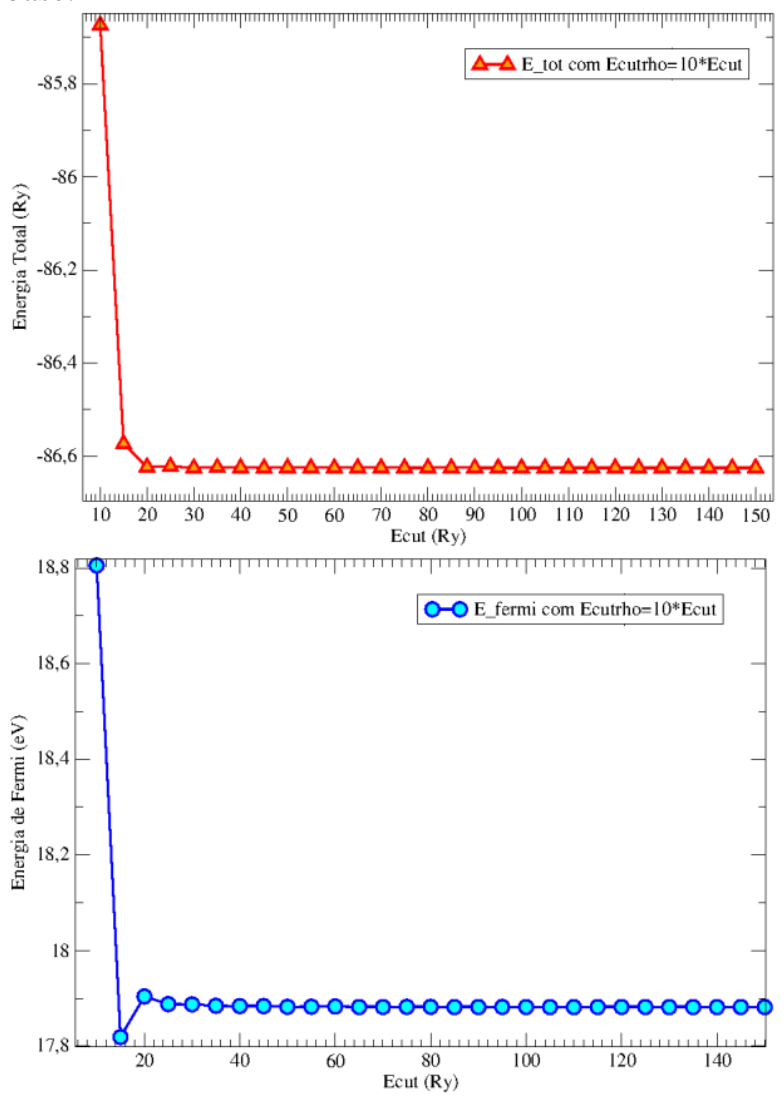

metodologias distintas para os obtenção de ao: (a) relaxação total da célula unitária (dados rotulados por Vc-relax na Tab.1); (b) vários cálculos de otimização de geometria com o parâmetro de rede fixo em valores que variavam entre $3.52 \AA$ a $4.32 \AA$ com passos de 0.01 Å e graficou-se a energia total em função do parâmetro de rede (ver Fig. 5) e identificou-se manualmente o ponto de mínimo na curva (dados rotulados por Avaliado na Tab.1) e (c) com os dados obtidos no passo (b) e mostrados na Fig. 5, graficou-se a energia total como função do volume do cristal e os dados foram ajustados por meio da equação de Bit- 
Murnaghan: $E(V)=E\left(V_{0}\right)+\frac{B_{0}}{B^{\prime}}\left[\frac{V_{0}\left(V_{0} / V\right)^{B^{\prime}-1}}{B_{0}^{\prime}-1}+\right.$

$\left.V\left(1-\frac{\left(V_{0} / V\right)}{B_{0}^{\prime}-1}\right)\right]$

onde $\mathrm{E}(\mathrm{V})$ representa o valor da energia do sistema em função do volume, $\mathrm{V}$ representa o volume, $\mathrm{V}_{0}$ é

Figura 5. Variação da energia total como função da variação dos parâmetros de redes do cristal de Pt.

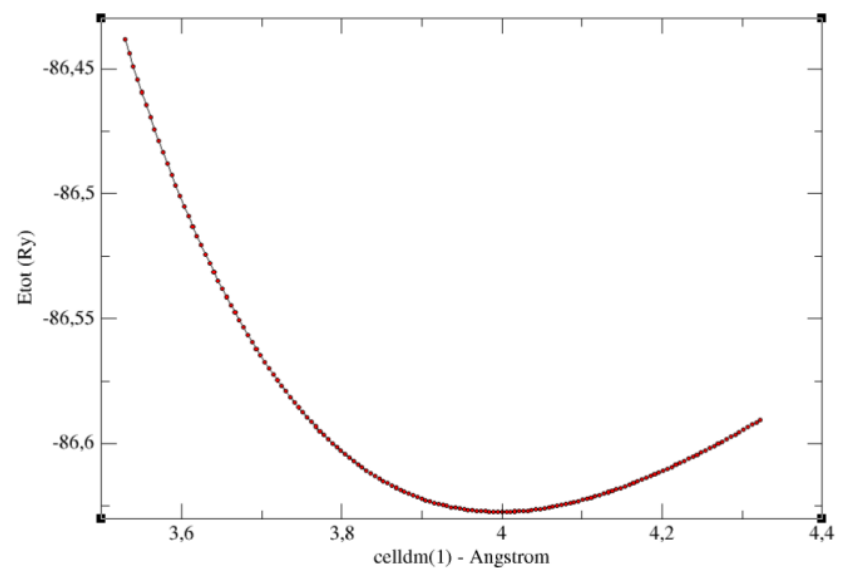

utilizado para volume de equilibrio, $\mathrm{E}\left(\mathrm{V}_{0}\right)$ é a energia mínima para o sistema, $B_{0}$ é denominado de módulo de compressibilidade e pode ser escrito como $B_{0}=$ $-V \frac{\partial P}{\partial V}=V \frac{d^{2} U}{d V^{2}}$ (lembrando-se que $\mathrm{dU}=-\mathrm{pdV}, \mathrm{T}=0$ $\mathrm{K})$. Assim, $\mathrm{B}_{0}$ é obtido por: $B_{0}(T, p)=-V \frac{\partial p}{\partial V}$. B' é a derivada do módulo de compressibilidade em relação à pressão.

Obteve-se a energia de coesão por meio da

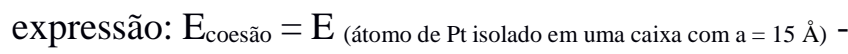

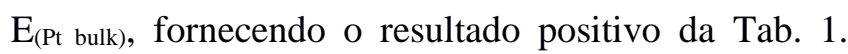
Observando os erros relativos, constata-se excelente acordo com dados experimentais e teóricos, indicando que a metodologia utilizada descreve bem o cristal de Pt.

A estrutura de bandas e a PDOS (Projeção da Densidade de Estados sobre os orbitais atômicos) calculadas para a Pt-bulk pode ser visualizada na Fig. 6. A PDOS foi calculada com uma grade de pontos muito mais densa (empregramos grade 60x60x60 e deslocado de Gamma). Em todos os casos o zero de energia foi deslocado para o valor da energia de Fermi. A contribuição dos estados d (em vermelho) e s (linha azul) foram separadas para facilitar a análise.

Figura 6. Estrutura de bandas para a Pt-bulk obtida com o funcional PBE. Inserimos ainda a DOS e PDOS calculadas (Density of States - Densidade de Estados) para comparação. $\mathrm{O}$ zero de energia foi deslocado para coincidir com o topo da banda de valência.

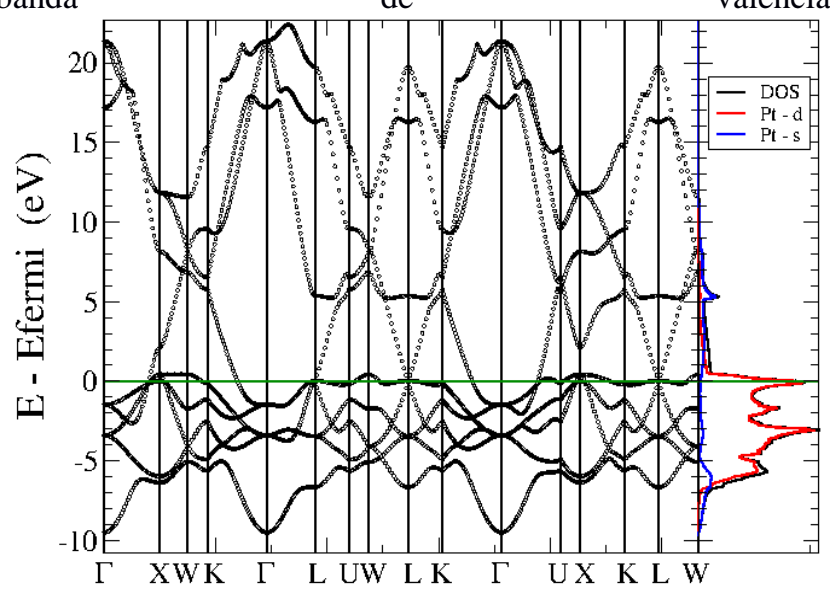

As características mostrados na na Fig. 6 estão em acordo com a literatura. O sistema é metálico (ver a linha verde posicionada no valor da energia de Fermi). Vê-se que o estado mais alto ocupado ora está abaixo da linha que representa a energia de Fermi, ora está acima. A DOS e a PDOS mostram que os estados eletrônicos próximos ao nível de Fermi têm caráter $\boldsymbol{d}$ e os estados de caráter $\boldsymbol{s}$ estão a $\sim 5 \mathrm{eV}$ abaixo do nível de Fermi.

A superfícies $\operatorname{Pt}(100)$ e $\operatorname{Pt}(111)$ foram modeladas computacionalmente empregando o modelo de fatias, consistindo de camadas de átomos de $\mathrm{Pt}$ separadas por um região de vácuo de $15 \AA$ e que tem por objetivo impedir a interação entre a fatia e sua imagem periódica em uma das direções espaciais, mas mantendo a periodicidade bidimensional característica das superfícies. A célula unitária para a direção cristalográfica (100) é tetragonal e com parâmetros de rede dados por $\frac{\sqrt{2}}{2} a \times \frac{\sqrt{2}}{2} a \times$ c.Para a direção cristalográfica (111), a célula unitária hexagonal tem parâmetros de rede $\frac{\sqrt{2}}{3} a \times \frac{\sqrt{2}}{3} a \times$ c. Em ambas 
estruturas, o parâmetro de rede c é a direção em que temos a camada de vácuo e a representa o parâmetro de rede do cristal de $\mathrm{Pt}$ (valores numéricos listados na Tab.

1). Foram construídas fatias contendo de 4 a 16 camadas atômicas. Imagens laterais e frontais para as fatias utilizadas para modelar as superfícies $\operatorname{Pt}(100)$ e
Pt (111) podem ser visualizadas na Fig. 7 e Fig. 8, respectivamente. $\mathrm{O}$ empilhamento de cada $\mathrm{ABAB}$ para a $\operatorname{Pt}(100)$ e ABCABC para a $\operatorname{Pt}(111)$ é evidenciado.

Tabela 1. Parâmetros de rede, energia de coesão bulk modulus calculados para o cristal de Pt com diferentes metodologias (ver texto para detalhes) e erros relativos comparados aos dados experimentais e teóricos das referências rotuladas por $*$ (SINGH-MILLER; MARZARI, 2009) e (SILVA; STAMPFL; SCHEFFLER, 2006) .

\begin{tabular}{llll}
\hline Pt - FCC & $\mathbf{a}_{\mathbf{0}}(\stackrel{\mathrm{A}}{)}$ & Erro Expt. $(\%)$ & Erro \\
\hline Vc-relax & 4,00386 & 2,1 & 0,3 \\
Fit (Murnaghan) & 3,99475 & 1,9 & 0,1 \\
Avaliado & 3,99475 & 1,9 & 0,1 \\
Expt.* & 3,92 & - & - \\
PBE* & 3,99 & - & -
\end{tabular}

\begin{tabular}{llll}
\hline Pt - FCC & $\mathbf{B}_{\mathbf{0}}(\mathbf{G P a})$ & Erro Expt. $(\%)$ & Erro Teor. $(\%)$ \\
\hline Fit (Murnaghan) & 260,3 & $-9,6$ & - \\
Expt. $*$ & 288 & - & - \\
PBE $*$ & 246 & Erro Expt. $(\%)$ & Erro Teor. $(\%)$ \\
\hline Pt - FCC & $\mathbf{E}_{\text {coesão }}(\mathbf{e V})$ & & 4,5 \\
PBE & $-5,84$ & 0,00 & - \\
PBE $* *$ & $-5,59$ & - & -
\end{tabular}

Testes de convergência de pontos k (ver Fig. 9) mostram que uma grade gerada com $\mathrm{k}_{\mathrm{x}} \times \mathrm{k}_{\mathrm{y}} \times \mathrm{k}_{\mathrm{z}}=12$ x 12 x 1 (deslocado) é adequado para o estudo das

Figura 7. Vista de topo (eixo $\mathrm{z}$ apontando para dentro da página) e lateral para a célula unitária utilizada para modelar a superfície $\operatorname{Pt}(100)$ transladada de nas direções $x$ e y.

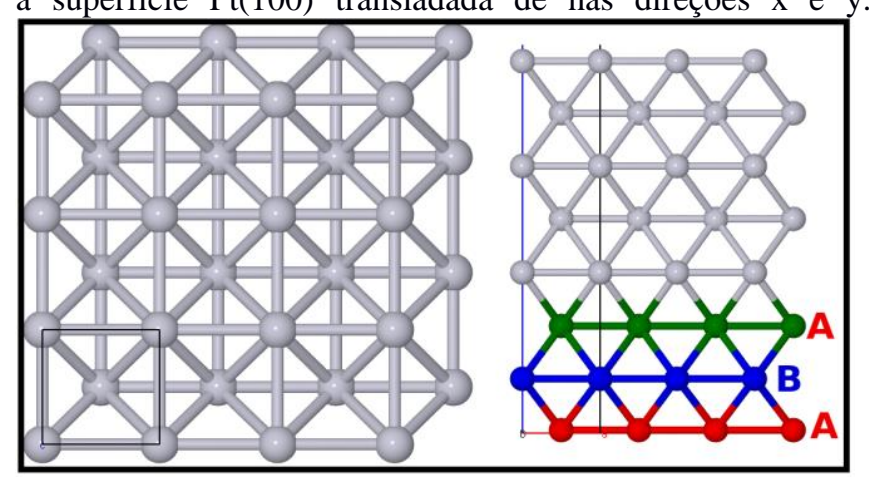

fatias. Cada um dos valores numéricos que aparecem no eixo $\mathrm{x}$ da Fig. 9 representa a grade de pontos $\mathrm{k}$ escolhida e não o número de pontos $\mathrm{k}$ utilizados na discretização do espaço recíproco, ou seja: o valor de $\mathrm{x}$ $=\mathrm{k}_{\mathrm{x}}$. Para citar um exemplo, $\mathrm{x}=4$ representa uma grade $4 \times 4 \times 1$ e assim por diante. 
Figura 8. Vista de topo (eixo $\mathrm{z}$ apontando para dentro da página) e lateral para a célula unitária utilizada para modelar a superfície $\operatorname{Pt}(111)$ transladada de nas direções $x$ e $y$.

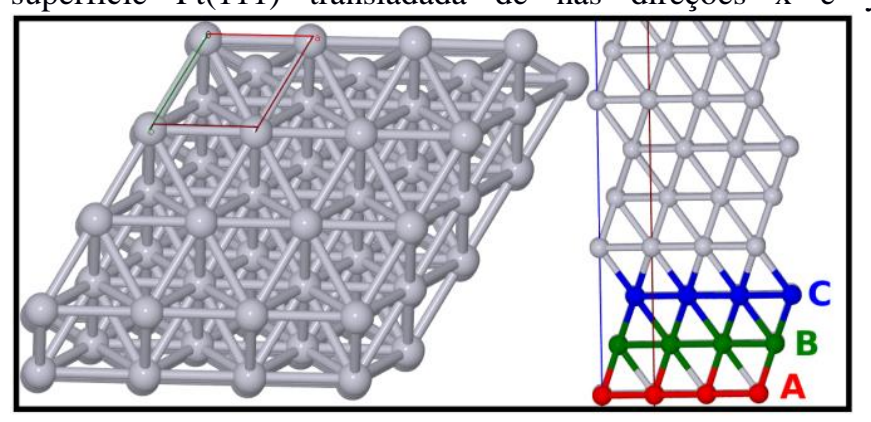

Átomos de Pt na superfície estão em ambiente diferente daqueles situados nas camadas internas e, em consequência, têm características distintas. Na Fig. 10 pode-se visualizar os valores das distâncias Pt-Pt para $\operatorname{Pt}(100)$ com diferentes números de camadas. Os valores se referem às distâncias interatômicas entre

Figura 9. Variação da energia total e da energia de Fermi para fatia de 8 camadas para modelagem da superfície $\operatorname{Pt}(100)$ como função da variação da grade pontos $\mathrm{k}$.
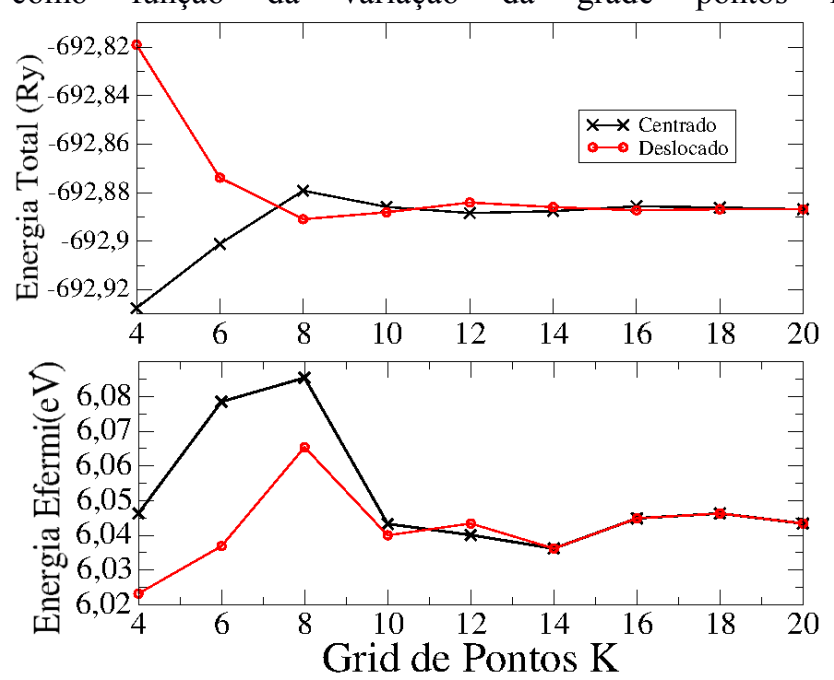

átomos posicionados em uma camada em relação a outro de uma camada imediatamente inferior. A linha contínua horizontal representa a distância $\mathrm{d}_{\mathrm{Pt}-\mathrm{Pt}}=2.831$ $\AA ̊$ do cristal. Ao lado do gráfico da Fig. 10, incluiu-se as distâncias para a fatia contendo 8 camadas atômicas e uma comparação com a superfície Pt(111) é mostrada na Fig. 11. A diferença entre $\mathrm{d}_{\mathrm{Pt}-\mathrm{Pt}}$ para os átomos de $\mathrm{Pt}$ da primeira e segunda camada e $\mathrm{d}_{\mathrm{Pt} \text {-Pt }}$ do cristal é de $0.041 \AA$ para $\operatorname{Pt}(100)$ e de apenas $0.009 \AA$ para
$\operatorname{Pt}(111)$. Estes resultados são consistentes com resultados teóricos e experimentais (KRUPSKI et al, 2015; SANTAROSSA et al, 2008) e descrevem corretamente a tendência de se obter uma contração da distância Pt-Pt para a superfície (100). Outro ponto a ser notado na Fig. 10 é que não há diferenças significativas para distâncias obtidas em fatias de 6,8 , 10, 14 e 16 camadas, fazendo com que sejam excelentes escolhas para simulação da superfície.

Figura 10. Distâncias interatômicas Pt-Pt para a superfície $\operatorname{Pt}(100)$ como função do número de camadas que compõem a fatia. A linha horizontal representa o valor da distância $\begin{array}{llll}\text { interatômica } & \text { para } & 0 & \text { cristal. }\end{array}$

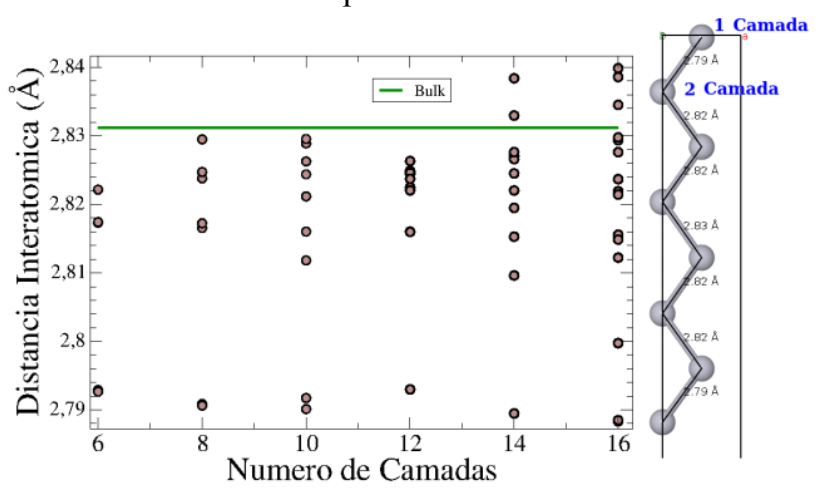


Figura 11. Distâncias interatômicas Pt-Pt para as superfícies $\operatorname{Pt}(100)$ e $\operatorname{Pt}(111)$ com 8 camadas atômicas.

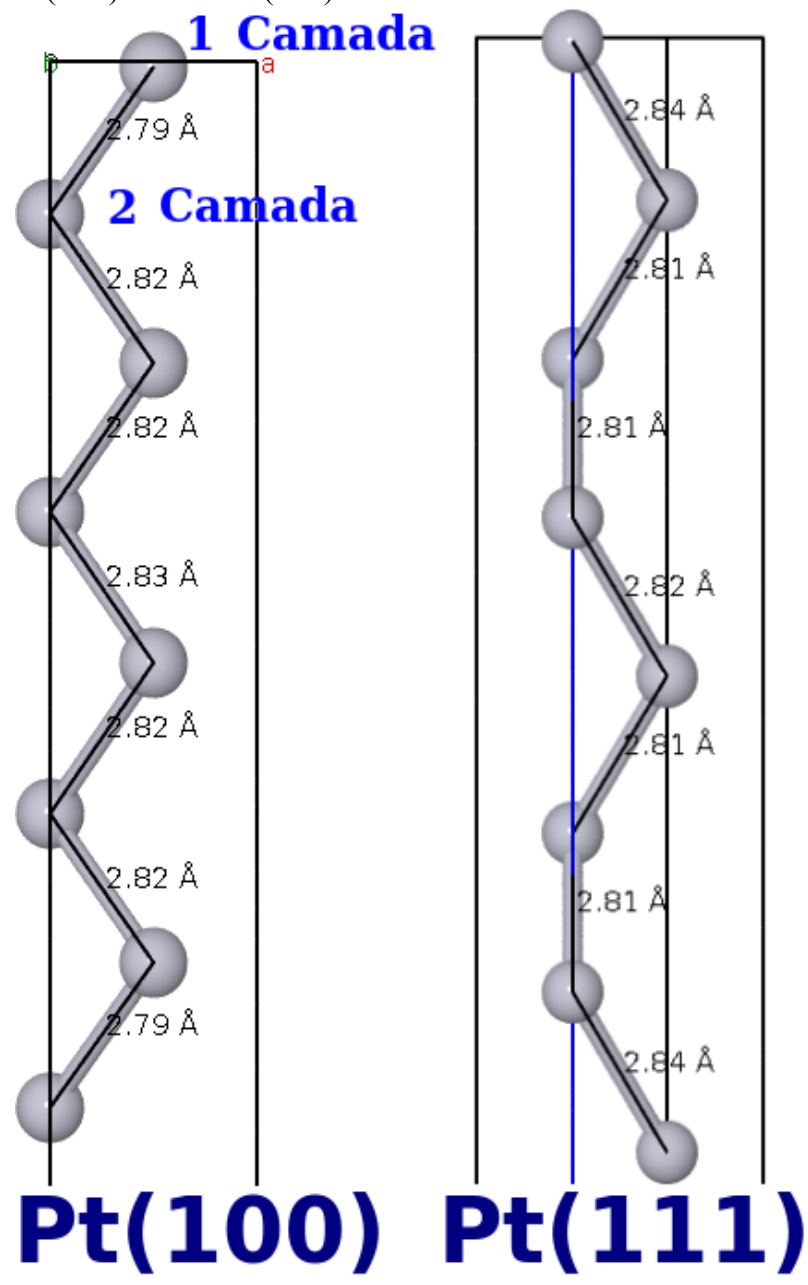

Na Tab. 2, resumiu-se os resultados de cálculos de energia de superfície, $\mathrm{E}_{\text {superficie }}(\mathrm{em} \mathrm{eV}$ por átomo), como função do número de camadas das fatias utilizadas para modelar computacionalmente as superfícies $\operatorname{Pt}(100)$ e $\operatorname{Pt}(111)$. E superficie foi calculada por meio da expressão: $\quad E_{\text {superficie }}=\frac{1}{2}\left(E_{\text {slab }}-\right.$ $N E_{\text {bulk }}$ ),onde $\mathrm{E}_{\text {slab }}$ representa a energia total de uma fatia, $E_{\text {bulk }}$ identifica a energia total do cristal de Pt e N o número de átomos que compõem a fatia. A energia de superfície está relacionada com a energia necessária para criar uma superfície (SINGH-MILLER; MARZARI, 2009) e, o fator $1 / 2$ foi incluído porque há 02 superfícies equivalentes por célula unitária. Há discussão na literatura sobre resultados diferentes para $\mathrm{E}_{\text {superficie }}$ se os cálculos para $\mathrm{E}_{\text {bulk }}$ forem realizados independentemente (SINGH-MILLER; MARZARI, 2009; SILVA; STAMPFL; SCHEFFLER, 2006; SANTAROSSA et al. 2008). Todavia, baseado nos resultados de (SILVA; STAMPFL; SCHEFFLER, 2006), não há diferenças significativas se todos os parâmetros forem bem convergidos. Assim sendo, os resultados apresentados foram obtidos a partir de $E_{\text {bulk }}$ calculada separadamente. Para comparação, incluiu-se na Tab. 2 dados experimentais (SINGH-MILLER; MARZARI, 2009) e teóricos (SILVA; STAMPFL; SCHEFFLER, 2006) com os quais há excelente acordo (ver erro relativo listado nas últimas 02 colunas). Constatou-se que $\mathrm{E}_{\text {superficie }}$ varia pouco com o aumento do número de camadas na fatia e indicam que fatias contendo entre 6 e 16 camadas são adequadas para descrever computacionalmente as superfícies investigadas. A informação é relevante porque no futuro pretende-se investigar caminhos de reação para a dissociação de etanol sobre Pt. Devido ao custo computacional elevado, será crucial reduzir o número de átomos do sistemas. 
Tabela 2. Energia de superficie para as fatias contendo diferente número de camadas de $\mathrm{Pt}$ (ver $\mathrm{N}_{\text {camadas }}$ ) utilizadas na modelagem computacional das superfícies Pt(100) e Pt(111). Dados experimentais (* - SINGH-MILLER; MARZARI, 2009 ) e teóricos (** - SILVA; STAMPFL; SCHEFFLER, 2006) foram incluidos para comparação.

\begin{tabular}{|c|c|c|c|c|c|c|}
\hline & & $\mathrm{E}_{\mathrm{s}}(\mathrm{eV} / \mathrm{at})$ & $\mathrm{E}_{\mathrm{s}}(\mathrm{eV} / \mathrm{at})^{*}$ & $\mathrm{E}_{\mathrm{s}}(\mathrm{eV} / \mathrm{at})^{* *}$ & Erro* & Erro** \\
\hline $\operatorname{Pt}(100)$ & 4 & 0,875 & - & 0,9 & - & 2,7 \\
\hline $\operatorname{Pt}(100)$ & 6 & 0,886 & - & 0,9 & - & 1,5 \\
\hline $\operatorname{Pt}(100)$ & 8 & 0,891 & - & 0,9 & - & 0,9 \\
\hline $\operatorname{Pt}(100)$ & 10 & 0,884 & - & 0,9 & - & 1,8 \\
\hline $\operatorname{Pt}(100)$ & 12 & 0,886 & - & 0,9 & - & 1,6 \\
\hline $\operatorname{Pt}(100)$ & 14 & 0,883 & - & 0,9 & - & 1,9 \\
\hline $\operatorname{Pt}(100)$ & 16 & 0,882 & - & 0,9 & - & 2,0 \\
\hline $\operatorname{Pt}(111)$ & 4 & 0,621 & 0,61 & 0,65 & $-1,8$ & 4,5 \\
\hline $\operatorname{Pt}(111)$ & 8 & 0,630 & 0,61 & 0,65 & $-3,3$ & 3,0 \\
\hline $\operatorname{Pt}(111)$ & 10 & 0,627 & 0,61 & 0,65 & $-2,8$ & 3,6 \\
\hline
\end{tabular}

Passando à análise das propriedades eletrônicas das fatias, apresenta-se, na Fig. 12, na Fig. 13 e na Fig. 14, a DOS e a PDOS para as superfícies $\operatorname{Pt}(100)$ e $\operatorname{Pt}(111)$.

Figura 12. Comparação entre a DOS para a superfície $\operatorname{Pt}(100)$ com 8 e 16 camadas e a superfície Pt (111) com 10 camadas. O zero de energia foi deslocado para o nível de Fermi de cada sistema.

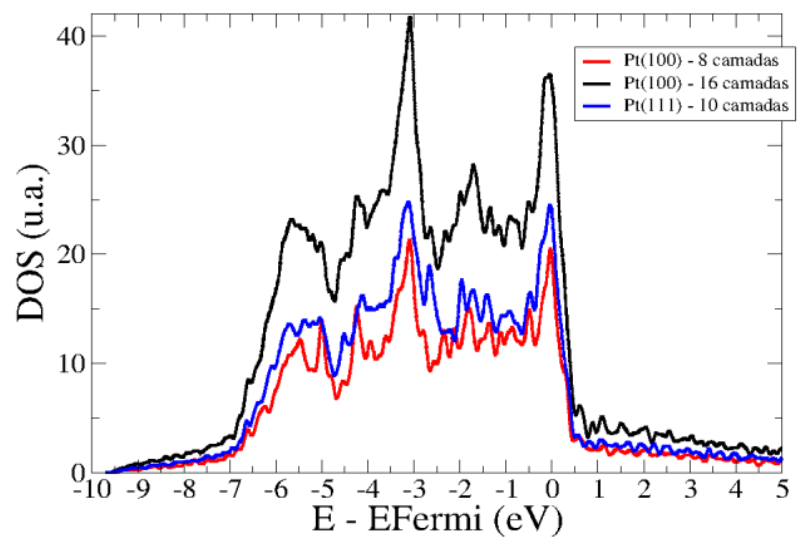

Figura 13. DOS para a superfície $\operatorname{Pt}(100)$ com 16 camadas. Inseriu-se a PDOS para os átomos de Pt da superfície (rotulados por camada 1), da segunda camada atômica (nomeados de camada 2) e pertencentes à terceira até a décima segunda camada atômica da fatia. O zero de energia foi deslocado para

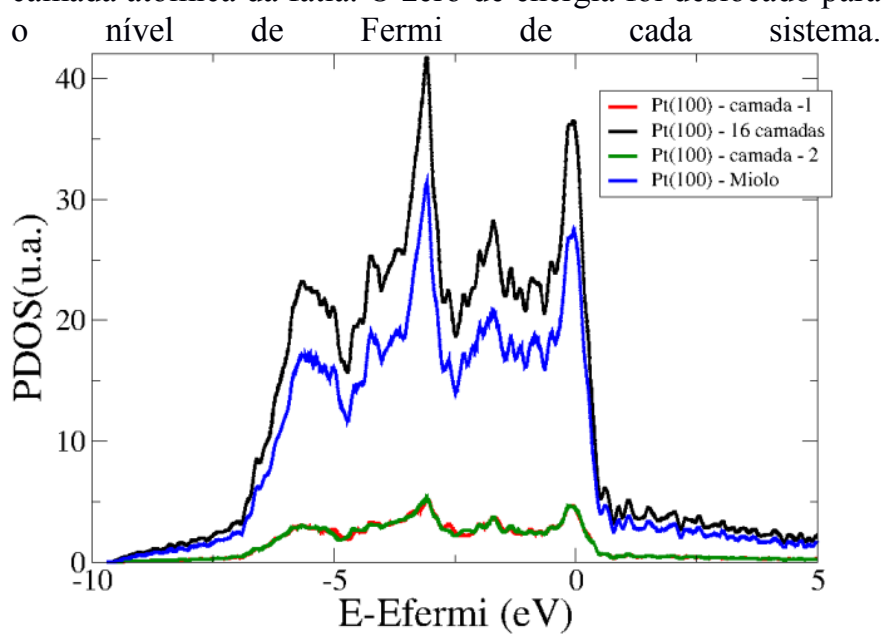

Em todos os gráficos, o zero de energia foi deslocado para coincidir com suas respectivas Energias de Fermi. Em todos os casos, os picos de DOS sobre a energia de Fermi indicam que as superfícies são metálicas e que tanto a Pt(100) quanto a Pt(111) têm 
DOS semelhantes (ver Fig. 12). De fato, não se nota característica marcante na DOS que possa ser usada para distinguir a $\operatorname{Pt}(100)$ da $\operatorname{Pt}(111)$.

Figura 14. DOS para a superfície $\operatorname{Pt}(111)$ com 10 camadas. Inseriu-se a PDOS para os átomos de $\mathrm{Pt}$ da superfície (rotulados por camada 1), da segunda camada atômica (nomeados de camada 2) e pertencentes à terceira até sexta camada atômica da fatia. O zero de energia foi deslocado para o nível de Fermi de cada sistema.

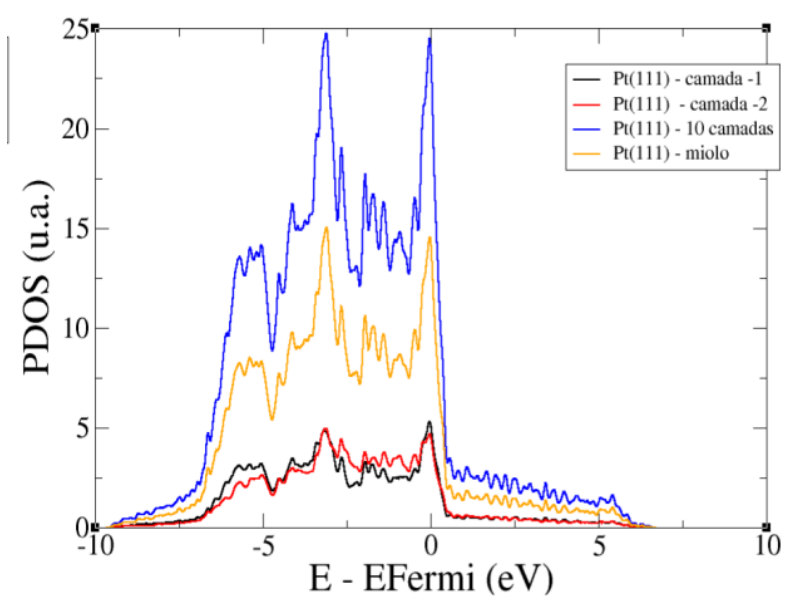

Em todos os gráficos, o zero de energia foi deslocado para coincidir com suas respectivas Energias de Fermi. Em todos os casos, os picos de DOS sobre a energia de Fermi indicam que as superfícies são metálicas e que tanto a $\operatorname{Pt}(100)$ quanto a $\operatorname{Pt}(111)$ têm DOS semelhantes (ver Fig. 12). De fato, não se nota característica marcante na DOS que possa ser usada para distinguir a $\operatorname{Pt}(100)$ da $\operatorname{Pt}(111)$.

Para investigar mais detalhes das propriedades eletrônicas, analisou-se a PDOS das diferentes regiões da fatia, como mostrado na Fig. 13 para a $\mathrm{Pt}(100)$ e na Fig. 14 para a $\operatorname{Pt}(111)$. Especificamente, graficou-se a PDOS para os átomos de Pt da superfície (rotulados por camada 1), daqueles pertencentes à segunda camada atômica (nomeados de camada 2) e para os demais átomos de Pt (rotulados por "miolo"). Nota-se que as diferentes regiões da fatia contribuem de forma semelhante para a DOS.

Apenas na Fig. 14 é que se nota algumas sutis diferenças entre átomos pertencentes à primeira e segunda camadas atômicas das fatias.
Comentou-se que um dos objetivos da investigação teórica deste trabalho era identificar o número mínimo de camadas que uma fatia deveria conter para representar adequadamente as propriedades das superfícies metálicas de Pt. Constatamos, por meio da análise da DOS (resultados não mostrados) que as fatias contendo apenas 04 camadas atômicas não são adequadas para modelar as superfícies porque surgem alguns "gaps" entre e desdobramentos de picos que não são observados em fatias com maior número de camadas. Uma das diferenças notáveis é o surgimento de 2 picos na região da Energia de Fermi.

\section{CONCLUSÃO}

Apresentou-se discussão detalhada das propriedades estruturais, energéticas e eletrônicas do cristal de Pt e as superfícies $\operatorname{Pt}(100)$ e $\operatorname{Pt}(111)$ obtidas por meio de simulações de primeiros princípios baseada na DFT, empregando pseudopotenciais e ondas planas como funções de base. Parâmetro de rede, Bulk Modulus e energia de coesão calculados para o cristal estão em excelente acordo com os dados experimentais e teóricos da literatura. Para as superfícies, a modelagem computacional se deu por meio do uso de fatias e investigou-se a influência do número de camadas nas propriedades das superfícies metálicas. Mostrou-se que fatias contendo pelo menos 6 camadas atômicas são necessárias para se descrever adequadamente estes sistemas. Alterações na DOS podem ser observadas quando se utiliza uma fatia composta por 4 camadas. Boa concordância com resultados teóricos e experimentais para energia de superfície mostram a robustez da metodologia proposta para investigação teórica das superfícies e evidenciam que esta pode ser adequada para modelagem de sistemas mais complexos envolvendo Pt. 


\section{AGRADECIMENTO}

Suporte financeiro do INEO-CNPq (Instituto Nacional de Eletrônica Orgânica) e o acesso aos recursos computacionais do CENAPADSP/UNICAMP (Centro de Processamento de Alto Desempenho de Campinas) foram fundamentais para o desenvolvimento deste trabalho.

Todos os autores declararam não haver qualquer potencial conflito de interesses referente a este artigo.

\section{REFERÊNCIAS}

CHUNG, D. Y. et al. Inhibition of co poisoning on pt catalyst coupled with the reduction of toxic hexavalent chromium in a dual-functional fuel cell. Scientific Rep., v. 4, n. 7450, p. doi:10.1038/srep07450, 2014.

GIANNOZZI , BARONI, P., S., BONINI, N., CALANDRA, M., CAR, R. , CAVAZZONI, C. , CERESOLI, D., CHIAROTTI, G. L., COCOCCIONI, M., DABO, I., DAL CORSO, A., DE GIRONCOLI, S., FABRIS, S., FRATESI, G., GEBAUER, R., GERSTMANN, U., GOUGOUSSIS, C., A. KOKALJ, M. LAZZERI, L. MARTIN-SAMOS, N. MARZARI, F. MAURI, R. MAZZARELLO, S. PAOLINI, A. PASQUARELLO, L. PAULATTO, C. SBRACCIA, S. SCANDOLO, SCLAUZERO, G., SEITSONEN, A. P. A. SMOGUNOV, P. UMARI e WENTZCOVITC, R. M.. QUANTUM ESPRESSO: a modular and opensource software project for quantum simulations of materials. Journal of Physics: Condensed Matter, v. 21, n. 39, p. 395502, 2009.

HOHENBERG, P. e KOHN, W. Inhomogeneous Electron Gas. Phys. Rev. B, v. 136, p. 864, 1964.

KOHN, W. e SHAM, L. J. Self-Consistent Equations Including Exchange and Correlations Effects. Phys. Rev., v. 140, p. A1133, 1965.

KORTLEVER, R., SHEN, SCHOUTEN, J. K. J. P., CALLE-VALLEJO, F. AND KOPER, M. T. M.. Catalysts and Reaction Pathways for the Electrochemical Reduction of Carbon Dioxide. J. Phys. Chem. Lett. v. 6, 4073, 2015.

KRUPSKI, K., MOORS, M., JÓŹWIK, P., KOBIELA, T. e KRUPSKI, A. Structure Determination of Au on
Pt(111) Surface: LEED, STM and DFT Study. Materials, v. 8, 2935, 2015.

LI, M.-R., CHEN, J. AND WANG, G.-C.. Reaction Mechanism of Ethanol on Model Cobalt Catalysts: DFT Calculations. J. Phys. Chem. C, v. 120, 14198, 2016.

LOPES., P. P. Aproveitamento de etanol em células a combustível: eletrocatálise da reação de oxidação direta e da oxidação do hidrogênio contaminado por co obtido por sua reforma. Tese de Doutorado-USP, São Carlos, 2013.

MARZARI, N.; VANDERBILT, D. Thermal contraction and disordering of the $\mathrm{Al}(110)$ surface. Phys Rev. Lett., v. 82, p. 3296, 1999.

PERDEW, J. P., BURKE, K. e ERNZERHOF, M. Generalized Gradient Approximation Made Simple. Phys. Rev. Lett., v. 77, p. 3865, 1996.

SANTAROSSA, G. VARGAS, A., IANNUZZI, M., PIGNEDOLI, C. A., PASSERONE, D. e BAIKER, A.. Modeling bulk and surface Pt using the "Gaussian and plane wave" density functional theory formalism: Validation and comparison to k-point plane wave calculations. The Journal Of Chemical Physics, v. 129, 234703, 2008.

SHENG, T., LIN, W-F., HARDACRE, C. and HU, P.. Role of Water and Adsorbed Hydroxyls on Ethanol Electrochemistry on Pd: New Mechanism, Active Centers, and Energetics for Direct Ethanol Fuel Cell Running in Alkaline Medium. J. Phys. Chem. C v. 118, 5762, 2014.

SILVA, J. L. D.; STAMPFL, C.; SCHEFFLER, M. Converged properties of clean metal surfaces by allelectron first-principles calculations. Surf. Sci., v. 600, p. 703-715, 2006.

SINGH-MILLER, N. E.; MARZARI, N. Surface energies, work functions, and surface relaxations of low-index metallic surfaces from first principles. Phys. Rev. B, v. 80, p. 235407, 2009.

SKÚLASON, E. TRIPKOVIC, V., BJORKETUN, M. E., GUDMUNDSDÓTTIR, S., KARLBERG, G., ROSSMEISL, J., BLIGAARD, T., JÓNSSON, H., AND NØRSKOV, J. K.. Modeling the Electrochemical 
Hydrogen Oxidation and Evolution Reactions on the Basis of Density Functional Theory Calculations. J. Phys. Chem. C, v. 114, 18182, 2010.

WENDT, H.; GöTZ, M.; LINARDI., M. Tecnologia de Células a combustível l. Quím. Nova., v. 23, n. 4, 2000.
ZHAO, L., WANG, S., DING, Q., XU, W., SANG, P., CHI, Y., LU, X. AND GUO, W.. The Oxidation of Methanol on PtRu(111): A Periodic Density Functional Theory Investigation. J. Phys. Chem. C, v. 119, 20389, 2015. 